\title{
UMA ABORDAGEM QUANTITIVA DO TEMA DE SUSTENTABILIDADE APRESENTADAS NO COBENGE NO PERÍODO DE 2010 A 2020
}

DOI: 10.37702/2175-957X.COBENGE.2021.3673

Ronaldo Florencio da Silva Junior - ronaldofjunior.14@gmail.com

Universidade Federal do Pará

Rua Dioniso Bentes 176

68795-000 - Benevides - PA

Luiz Eduardo Sousa Sena - luiz.sena@itec.ufpa.br

UFPA

Rua Rio Branco 376

66617-080 - Belém - PA

ATHUS IGOR CASTRO HOLANDA - athusholanda07@gmail.com

UNIVERSIDADE FEDERAL DO PARÁ

CONJUNTO STÉLIO MAROJA, WE 1, QUADRA B, BLOCO 1106

67140-380 - ANANINDEUA - PA

Marcos Silvio Borges Inete - marcos.inete@itec.ufpa.br

Universidade Federal Do Pará

Porto do Arapari 06

68445-000 - Barcarena - PA

Gustavo Lisboa Negrão - gustavo.negrao@itec.ufpa

Universidade Federal do Pará

Rodovia Augusto Montenegro 1663

66635-110 - Belém - PA

MARIA DE FATIMA MENDES LEAL - fa.leal@gmail.com

Universidade Federal do Pará

Avenida Gov Magalhães Barata, 110

66060-281 - Belém - PA

Resumo: O Congresso Brasileiro de Educação em Engenharia (COBENGE) é um 
dos maiores eventos sobre o ensino de engenharia do Brasil, realizado pela Associação Brasileira de Educação em Engenharia (ABENGE), anualmente desde 1973. Este artigo apresentará um panorama do que se publicou sobre o tema sustentabilidade, uma das subáreas do evento, nos últimos 10 anos. Foram analisados 46 artigos entre 2010 e 2020, exceto o ano de 2013 e 2015 por indisponibilidade dos anais no site da ABENGE. Os resultados mostram que $o$ ano de 2014 (9) e 2020 (7) foram os que mais houveram publicações sobre o tema sustentabilidade; as instituições que mais desenvolveram artigos sobre a temática foi a UFPA (4), USP (4) e o CEFET-MG (4), todas possuem programas de pós-graduação em desenvolvimento sustentável e/ou meio ambiente; através da nuvem de palavras se constatou que os termos mais utilizados foram: engenharia, educação, desenvolvimento, ensino e sustentabilidade, o que já era esperado se tratando do maior evento de ensino do país; por meio do modelo Triple Bottom Line foi possível demonstrar que os trabalhos seguiam pelo menos um dos três pilares da sustentabilidade: o social, o ambiental e o econômico; das regiões brasileiras a que mais desenvolveu estudos sobre o tema foi a região sudeste, representando sozinha $45,7 \%$ dos trabalhos publicados no evento sobre sustentabilidade. Com base nos resultados foi possível destacar o valor e a contribuição do congresso para impulsionar o debate sobre a engenharia sustentável, formação cidadã e responsabilidade social e institucional.

Palavras-chave: Sustentabilidade. Educação. COBENGE 


\section{UMA ABORDAGEM QUANTITIVA DO TEMA DE SUSTENTABILIDADE APRESENTADAS NO COBENGE NO PERÍODO DE 2010 A 2020}

\section{INTRODUÇÃO}

As crises ambientais, mudanças climáticas e o risco a vida na terra têm feito a humanidade procurar meios de se desenvolver de forma sustentável, em harmonia com o meio ambiente para evitar novas crises e amenizar as que já estão em curso. O desafio desta geração é usufruir dos recursos naturais presentes no planeta sem comprometer o uso das gerações futuras. Dentro desse contexto as engenharias surgem como protagonistas no debate sobre novos meios de produção, novos materiais, novas técnicas, melhoramento de processos e na geração de conhecimento aliado ao desenvolvimento sustentável.

O conhecimento é produzido diariamente, porém o avanço somente ocorre com a exposição de ideias e com o debate técnico adequado. Com objetivo de fomentar e qualificar o debate acadêmico, a Associação Brasileira de Educação em Engenharia (ABGENGE) promove anualmente o Congresso Brasileiro de Educação em Engenharia (COBENGE) desde 1973, O evento tem como objetivo contribuir para a melhoria do ensino e o exercício da engenharia por meio do debate e do compartilhamento de conhecimentos e, consequentemente, ampliando os benefícios que a engenharia pode proporcionar a toda a sociedade. O Congresso possui atualmente 14 áreas e 56 subáreas. A área de formação cidadã e sua respectiva subárea sustentabilidade se propõem a debater temas relacionados ao meio ambiente e sua importância para o desenvolvimento sustentável.

A sustentabilidade, nos últimos anos, tem sido o tema central de vários estudos. Sendo assim o objetivo este artigo é a verificação de como o tema da sustentabilidade tem sido tratado, por meio da análise quantitativa do que foi publicado no COBENGE na última década. Foram considerados os trabalhos apresentados no período de 2010 a 2020. Os trabalhos foram classificados por: ano de publicação, região do brasil, instituição e título.

\section{REFERENCIAL TEÓRICO}

Conforme o Artigo 225 da Constituição Federal de 1988, "todos têm direito ao meio ambiente ecologicamente equilibrado, bem de uso comum do povo e essencial à sadia qualidade de vida, impondo-se ao poder público e à coletividade o dever de defendê-lo e preservá-lo para as presentes e futuras gerações". Sabendo-se que sustentabilidade pode ser entendida como a consequência de princípios básicos da ecologia, tais como, interdependência, reciclagem, parceria, flexibilidade e diversidade (CAPRA, 2006).

Considerando que a engenharia é o campo profissional, prático e teórico, que relaciona a aquisição e aplicação de conhecimento técnico científico com a invenção, inovação e uso de materiais, métodos e processos para fins específicos (Unesco Report, 2010), pode-se deduzir que, desenvolvimento sustentável se baseia na adaptação das práticas dentro da metodologia de trabalho das engenharias às fortes necessidades que 0 mundo desenvolveu com relação à conservação dos recursos naturais para que estes possam perpassar as gerações. Nem sempre a sustentabilidade foi uma grande preocupação no âmbito da engenharia. Hoje, já existem estratégias que buscam romper com os currículos disciplinares tradicionais que apresentam maiores limitações ao tratar de questões centrais de formação que requer olhar integrado e compreensão da complexidade das questões ambientais (ROTTA; BATISTELA; FERREIRA, 2017). Hoje se faz necessário 
que exista uma ponte entre os trabalhos relacionados à engenharia e o desenvolvimento sustentável, que possuam uma consciência ambiental, além de eficiência técnica e científica. Moreira e Candau (2006) definem o currículo como um objeto de reflexão variada, que se organizará de acordo com os sujeitos e o espaço que o envolve, é evidente que ao passo em que as circunstâncias ambientais passam a exigir mudanças voltadas à sustentabilidade, é natural que os currículos se adaptem a esta realidade. A missão do COBENGE, é a de "produzir mudanças necessárias para a melhoria da qualidade do ensino de graduação e pós-graduação em engenharia e tecnologia no Brasil, contribuindo para a formação de profissionais cada vez mais qualificados, promovendo desenvolvimento e tecnologia a todos os pontos do país pelos benefícios que a engenharia pode proporcionar a toda população" (ABENGE, 2020). Portanto, o tema sustentabilidade é um assunto que deve, cada vez mais, ser abordado sob diversos aspectos nos eventos COBENGE evidenciando a relação entre a engenharia e a sustentabilidade. Considerando o exposto, esse trabalho se justifica ao traçar um panorama do que se publicou sobre sustentabilidade na última década do COBENGE. Esta análise servirá como um indicador para demostrar a relevância do evento ao fomentar o debate ambiental no Brasil.

\section{METODOLOGIA}

O presente trabalho se constitui de uma pesquisa bibliográfica de caráter quantitativo nos anais do Congresso Brasileiro de Educação em Engenharia - COBENGE, publicados entre os anos de 2010 a 2020. O estudo abrangeu a área de formação cidadã e subárea sustentabilidade, uma das 56 subáreas do evento.

Por meio do filtro disponível no site da Associação Brasileira de Educação em Engenharia - ABENGE foi possível separar os artigos referentes ao tema sustentabilidade apenas entre os anos de 2017 a 2020. Nos anos anteriores, por diferença de interface, o site não permitiu usar o mesmo filtro sendo necessário usar como palavras chaves os termos sustentabilidade, sociedade, meio ambiente, lixo e resíduos sólidos a fim de selecionar os artigos entre 2010 e 2016. Estas palavras foram selecionadas com base na leitura dos artigos filtrados entre 2017 e 2020, tendo em consideração que os artigos apresentaram pelo menos uma vez essas palavras. Cabe ressaltar que os anos de $2013 \mathrm{e}$ 2015 foram excluídos dessa revisão por não estarem disponíveis no site da ABENGE, o que impossibilitou a análise dos anais desses 2 anos.

$\mathrm{Na}$ análise dos dados, foi feita a extração e compilação dos artigos no Excel da Microsoft. O estudo se limita aos seguintes tópicos: ano de publicação, instituições, palavras-chave, pilares da sustentabilidade abordados através do modelo Triple Bottom Line e região do estudo.

Na primeira etapa do estudo foi realizado a leitura do tema, resumo, palavras-chave e resultados dos artigos selecionados. A segunda etapa consistiu em organizar os dados na planilha eletrônica seguindo a ordem de: ano de publicação, instituição, título e os pilares da sustentabilidade abordados. Mediante os dados levantados foi possível criar gráficos, e tabelas que explicitam os resultados encontrados. A última etapa deste estudo foi a análise, discussão e construção dos resultados. 


\section{RESULTADOS}

\subsection{0 anos sobre o tema sustentabilidade no COBENGE}

O número de trabalhos no período selecionado foi de 46 artigos. O ano de 2014 foi o ano com a maior quantidade de artigos publicados dentro do tema sustentabilidade, representando $20 \%$ do total estudado. Os anos de 2010 e 2012, foram apresentados somente $7 \%$ do total sobre o tema sustentabilidade. Vale ressaltar que nos anos 2010 e 2012 os temas do evento foram "engenharia em movimento" e "o engenheiro professor e o desafio de educar", respectivamente. Nesses anos os artigos estiveram mais voltados a importância do tema sustentabilidade no currículo das faculdades de engenharia no brasil.

No ano de 2014 o evento trouxe o tema "engenharia: múltiplos saberes e atuações" e os artigos estavam mais voltados ao tema desenvolvimento econômico sustentável e os impactos sociais, conforme Figura. 1.

Figura 1 - Publicações sobre sustentabilidade no

COBENGE entre 2010 e 2020, exceto 2013 e 2015.

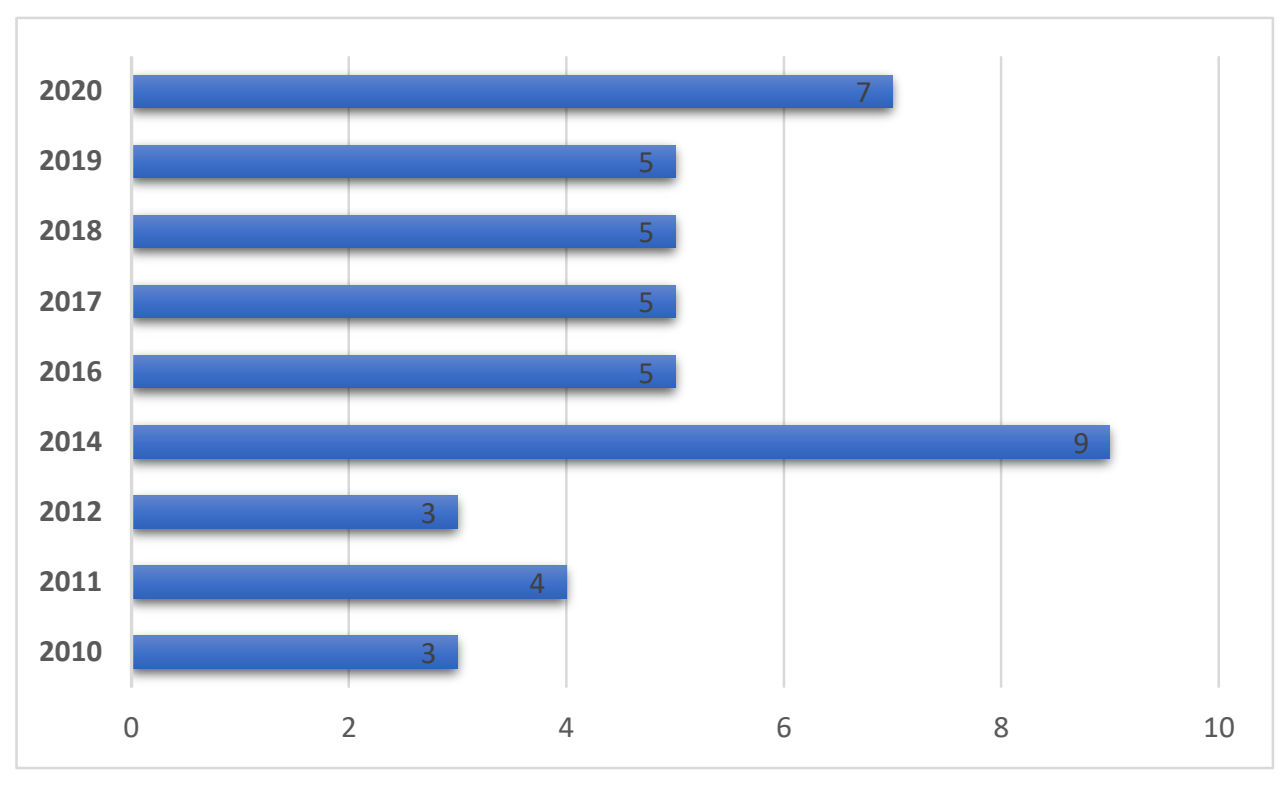

Fonte: Dados da pesquisa (2021)

\subsection{Instituições}

Nos últimos 10 anos, 32 instituições diferentes publicaram no COBENGE sobre o tema sustentabilidade e suas variações. As instituições com a maior quantidade de publicações foram: Universidade Federal do Pará (UFPA), Universidade Tecnológica Federal do Paraná (UFTPR), Universidade de São Paulo (USP) e o Centro Federal de Educação Tecnológica de Minas Gerais (CEFET/MG), todas com quatro publicações cada. A Universidade Federal de Santa Catarina (UFSC) e a Pontifícia Universidade Católica de Goiás (PUC/GO) apresentaram três trabalhos cada. As Universidade Federal Fluminense e Centro Federal de Ensino Tecnológico do Rio de Janeiro (CEFET/RJ) apresentaram dois trabalhos cada, conforme Figura 2. 
Figura 2 - As 8 Instituições que mais publicaram sobre sustentabilidade entre 2010 e 2020

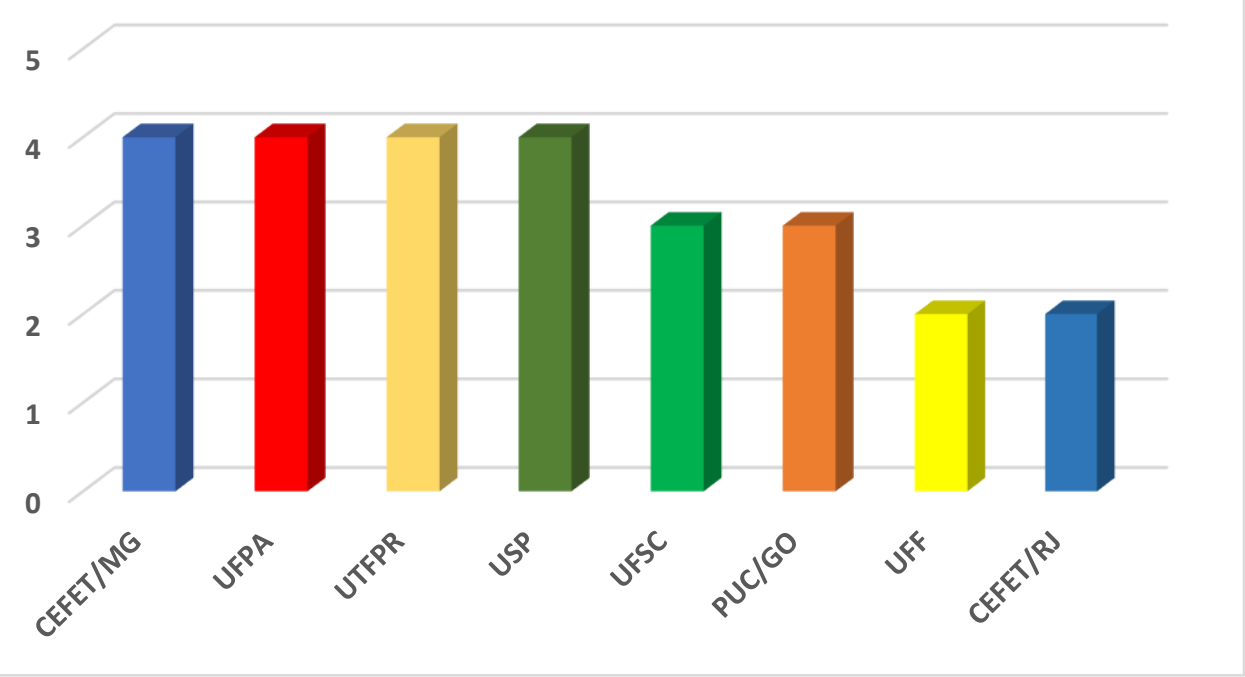

Fonte: Dados da pesquisa (2021)

As quatro instituições com as maiores quantidades de publicações sobre o tema ambiental possuem programas de pós-graduação em pelo menos uma das áreas relacionadas ao tema sustentabilidade. A Universidade Federal do Pará possui o Programa de Pós-Graduação em Ciências e Meio Ambiente (PPGCMA), criado em 2012 e vinculado ao Instituto de Ciências Exatas e Naturais da UFPA e tem como objetivo formar profissionais capacitados para atuar em questões relacionadas ao meio ambiente. Na mesma linha, a USP possui um programa específico de pós-graduação em sustentabilidade criado em 2004.O CEFET-MG e a UFTPR possuem programas dentro das linhas de sustentabilidade de processos controle ambiental e sustentabilidade ambiental urbana, respectivamente.

\subsection{Palavras chaves e nuvem de palavras}

As palavras chaves representam, de alguma forma, o conteúdo dos artigos. Assim sendo, detectou-se que nos 46 artigos analisados foram identificadas 254 palavras-chave, entre as quais engenharia, educação, desenvolvimento, ensino, sustentabilidade e sustentável foram as mais frequentes. Considerando a missão do COBENGE, que é um dos maiores congressos de ensino em engenharia do Brasil, verifica-se a preocupação dos autores com a educação e a engenharia e sua aplicação nos diversos temas do Congresso.

\subsection{Pilares da sustentabilidade abordados}

Observou-se que nos artigos pesquisados houve uma concentração nos pilares da sustentabilidade representados pelos indicadores econômico, ambiental e social.

$\mathrm{Na}$ linha econômica se analisou os trabalhos referentes a melhoria e desenvolvimento sustentável de novos produtos/processos com objetivo de aumentar os lucros sem agredir o meio ambiente. Na linha ambiental se observou as publicações relacionadas a reciclagem, reutilização e impactos ao meio ambiente. Na linha social se examinou artigos pautados em responsabilidade social, impactos na comunidade, interação social e na formação cidadã dos futuros profissionais. A ênfase no tripé da sustentabilidade pode ser verificada na Figura 3. 
Figura 3 - Artigos por pilares da sustentabilidade nos anos de 2010 a 2020

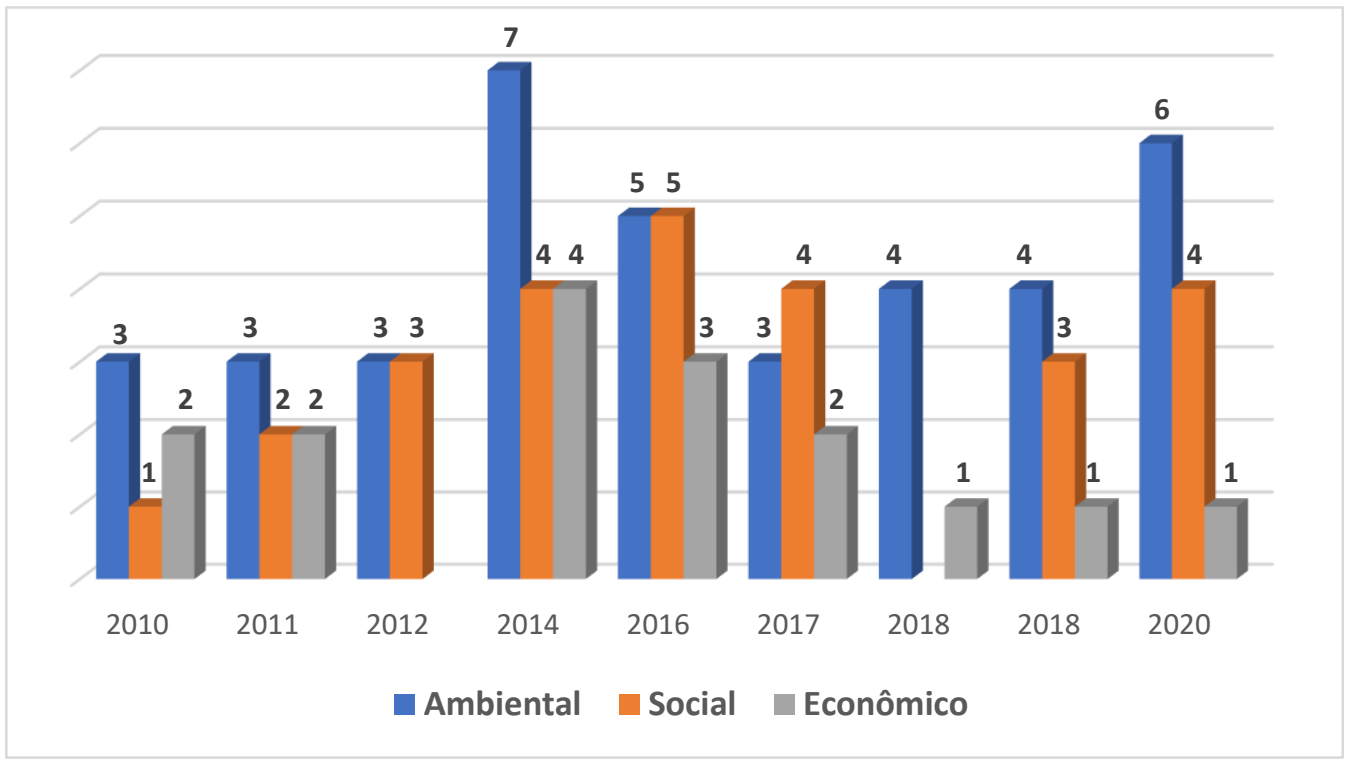

Fonte: Dados da pesquisa (2021)

Considerando os trabalhos que seguiam apenas um dos três pilares da sustentabilidade verificou-se que o tripé mais abordado foi tripé ambiental com oito artigos seguido pelo social com quatro publicações e o econômico com dois artigos. A maioria das publicações seguiu pelo menos duas linhas ou as três ao mesmo tempo: social e ambiental (17 artigos), ambiental e econômico com cinco artigos, econômico e social um artigo. O uso dos indicadores econômico, social e ambiental, ao mesmo tempo, foi comtemplado em sete artigos. Conclui-se, portanto, que a maioria das publicações abrangeram pelo menos dois dos três pilares da sustentabilidade.

\subsection{Regiões que mais publicaram sobre sustentabilidade}

Como o COBENGE abrange publicações de diversas engenharias em todo o Brasil, definiu-se um panorama sobre as regiões do país que mais publicaram sobre o tema nos últimos dez anos do evento. A figura 4, a seguir, ilustra o quantitativo em termos percentuais. 
Figura 4 - Regiões do Brasil que mais publicaram no COBEGNGE sobre sustentabilidade

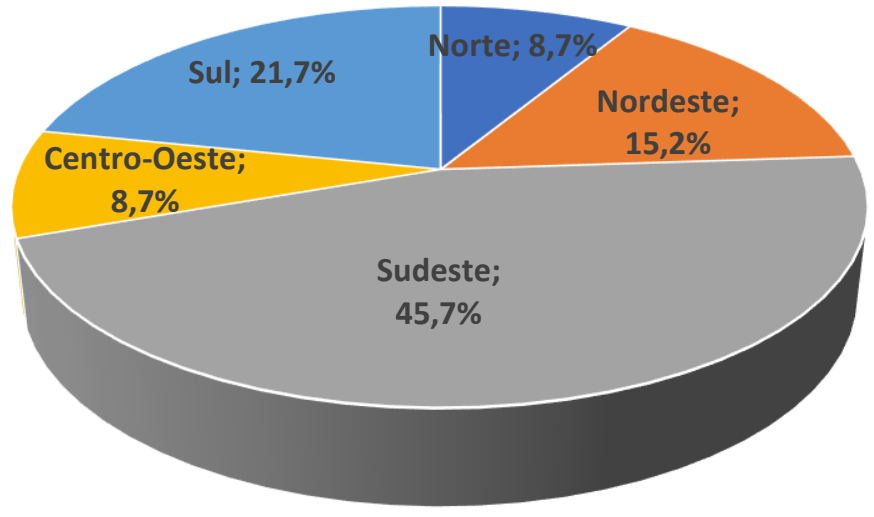

Fonte: Dados da pesquisa (2021)

A região com a maior quantidade de trabalho sobre o tema foi a região Sudeste com $45,7 \%$ das publicações (21 artigos). A segunda região com mais publicações foi a Sul com $21,7 \%$ (10 artigos), seguida por Nordeste com 15,2\% com sete artigos e Centro-Oeste e Norte ambas com 8,7\% com quatro artigos cada.

\section{CONSIDERAÇÕES FINAIS}

Levando-se em conta o que foi observado a partir da análise dos 46 artigos publicados nos últimos 10 anos do COBENGE, conclui-se que o tema sustentabilidade sempre esteve presente nos trabalhos apresentados. Em alguns momentos o tema foi mais abordado, como no ano de 2014 e 2020, quando forma apresentados mais publicações sobre o assunto. Nos anos de 2010 e 2020, o tema sustentabilidade foi menos divulgado. O estudo evidencia a importância das publicações no Congresso cujos resultados e conclusões servem para fomentar a discussão sobre uma engenharia moderna e sustentável, que se preocupa com outros fatores que vão muito além de custo e prazo.

As instituições que mais tiveram trabalhos publicados foram exatamente as que possuem programas de pós-graduação específicos sobre o tema sustentabilidade, destacase o CEFET-MG com o programa sustentabilidade de processos e controle ambiental. A UFPA oferece um programa na mesma linha, o de pós-graduação em ciência e meio ambiente com objetivo de formar profissionais preparados para tratar com questões relacionadas ao setor ambiental. Por meio deste estudo foi possível constatar a grande relevância das universidades como formadoras de opinião e geradoras de conhecimento para a construção de uma sociedade com mais consciência para a importância do desenvolvimento sustentável.

Por meio dos termos das palavras-chave a presença de "ensino" e "engenharia", por exemplo servem de indicadores da importância do Cobenge para o ensino em engenharia no Brasil. O termo sustentabilidade também foi muito utilizado, revelando que o evento tem buscado cada vez mais unir o ensino da engenharia ao desenvolvimento sustentável. 
Uma das dificuldades para a realização deste trabalho sobre o um estudo das publicações dos últimos 10 anos do evento, vale a penas salientar algumas limitações, tais como a ausência de análise dos anais dos anos de 2013 e 2015, o que se considerado, poderia evidenciar ainda mais a importância do congresso em promover o debate ambiental. Os resultados obtidos nesse trabalho trazem uma visão geral do que se publicou na última década no COBENGE sobre sustentabilidade e meio ambiente e tais resultados podem servir para evidenciar o valor e a contribuição do congresso para impulsionar o debate sobre a engenharia sustentável, formação cidadã e responsabilidade social e institucional.

\section{REFERÊNCIAS}

ABENGE. Congresso Brasileiro de Educação em Engenharia 2020. Disponível em: http://www.abenge.org.br/cobenge/2020/. Acesso em 06/05/2021

BRASIL. Constituição (1988). Constituição da República Federativa do Brasil. Brasília, DF: Senado Federal: Centro Gráfico, 1988.

CAPRA, Fritjof. As conexões ocultas: ciência para uma vida sustentável. São Paulo: Cultrix, 2005.

MOREIRA, A. F.; CANDAU, V. M. Indagações sobre currículo: currículo, conhecimento e cultura. Brasília: Ministério da Educação, Secretaria de Educação Básica, 2006

ROTTA, Mariza; BATISTELA, Airton Carlos; FERREIRA, Sergio Ricardo. Ambientalização curricular no Ensino Superior: Formação e Sustentabilidade nos Cursos de Graduação. Actualidades Investigativas en Educación, [s. I.], v. 17, n. 2, p. 12, 2017.

UNESCO. UNESCO Science Report, 2010. Disponível em: http://www.unesco.org/new/en/natural-sciences/science-technology/prospectivestudies/unesco-science-report/unesco-science-report-2010/ Acesso em: 03/05/2021.

\section{A QUANTITIVE APPROACH TO THE SUSTAINABILITY THEME PRESENTED AT COBENGE IN THE PERIOD FROM 2010 TO 2020}

Abstract: The Brazilian Congress of Engineering Education (COBENGE) is one of the largest events on engineering education in Brazil, held by the Brazilian Association of Engineering Education (ABENGE), annually since 1973. This article will present an overview of what has been published on the theme of sustainability, one of the subareas of the event, in the last 10 years. 46 articles were analyzed between 2010 and 2020, except the year 2013 and 2015 due to unavailability of annals on the ABENGE website. The results show that 2014 (9) and 2020 (7) were the most published publications on the topic of sustainability; the institutions that most developed articles on the theme were UFPA (4), USP (4) and CEFET-MG (4), all have graduate programs in sustainable development and / or the environment; through the word cloud it was found that the most used terms were: 
engineering, education, development, teaching and sustainability, which was already expected as it is the largest educational event in the country; through the Triple Bottom Line model, it was possible to demonstrate that the works followed at least one of the three pillars of sustainability: the social, the environmental and the economic; of the Brazilian regions the one that most developed studies on the theme was the southeast region, representing $45.7 \%$ of the works published in the event on sustainability alone. Based on the results, it was possible to highlight the value and contribution of the congress to stimulate the debate on sustainable engineering, citizen training and social and institutional responsibility.

Keywords: Sustainability. Education. COBENGE 